\title{
A reinvestigation of the synthesis of 1-aminoarylmethylphosphonates on the surface of alumina and novel method for the synthesis of bis[1-diethoxyphosphoryl aryl methyl] amines
}

\author{
Babak Kaboudin, ${ }^{* a}$ Khavar Moradi, ${ }^{a}$ and Ali Reza Sardarian ${ }^{b}$ \\ ${ }^{a}$ Department of Chemistry, Institute for Advanced Studies in Basic Sciences (IASBS), Gava Zang, \\ Zanjan 45195-1159, Iran \\ ${ }^{b}$ Department of Chemistry, Faculty of Science, Shiraz University, Shiraz, Iran \\ E-mail: kaboudin@iasbs.ac.ir
}

\begin{abstract}
In 1997 we published a simple and efficient method for the synthesis of 1aminoarylmethylphosphonates from one-pot reaction aromatic aldehydes, hexamethyldisilazane and diethylphosphite. ${ }^{1}$ In 2003 Soroka and Kolodziejczyk ${ }^{2}$ published comments on this work and they believed that aromatic aldehydes react with diethyl phosphite and hexamethyldisilazane to give 1-(trimethylsilyloxy)-1-arylmethylphosphonates instead of 1-amino-1-arylmethylphosphonate. Therefore we decided to analyze and reinvestigate this reaction and also novel method for the synthesis of bis[1-diethoxyphosphorylarylmethyl] amines using of acetyl chloride as catalyst in hydrophosphonylation of imine is described.
\end{abstract}

Keywords: Phosphonic acids, amino acids, alumina, aldehydes, diimines, addition reaction

\section{Introduction}

Organophosphorus compounds have found a wide range of applications in the areas of industrial, agricultural, and medicinal chemistry owing to their biological and physical properties as well as their utility as synthetic intermediates. ${ }^{3} \alpha$-Functionalized phosphonic acids are valuable intermediates for the preparation of medicinal compounds and synthetic intermediates. ${ }^{4}$ Among $\alpha$-functional phosphonic acids, 1-aminophosphonic acids are an important class of compounds that exhibit a variety of interesting and useful properties. The 1-aminophosphonic acids are the most important substitutes for the corresponding amino acids in biological systems. ${ }^{5,6}$ Indeed a number of potent antibiotics, ${ }^{7}$ enzyme inhibitors, ${ }^{8}$ and pharmacological agents ${ }^{9}$ contain 1 aminophosphonic acids as well as their derivatives, notably peptides. These important compounds have been synthesized by various routes: (a) addition of P-H function to imines and 
enamines, ${ }^{10}$ (b) addition of P-H function to nitriles, ${ }^{11}$ (c) Arbuzov and Michaelis-Becker reactions, ${ }^{12}$ (d) condensation of $\mathrm{X}-\mathrm{NH}_{2}$ with acyl phosphorus species, ${ }^{13}$ (e) Curtius and Hofmann rearrangement of substituted phosphonoacetic esters, ${ }^{14}$ and (f) alkylation of nucleophilic precursors such as Schiff bases. ${ }^{15}$

Surface-mediated solid phase reactions are of growing interest ${ }^{16}$ because of their ease of set up and work-up, mild reaction conditions, rate of reaction, selectivity, high yields, lack of solvent and the low cost of the reactions in comparison with their homogeneous counterparts. In 1997 we published unexpected results on the synthesis of 1-aminoarylmethylphosphonates in the reaction of aromatic aldehydes, hexamethyldisilazane (HMDS) and diethyl phosphite, via diethyl N-arylidene-1-amino-1-arylmethylphophonate on the alumina surface. In 2003 Soroka and Kolodziejczyk published comments on this reaction. They found aromatic aldehydes react with diethyl phosphate and HMDS to give 1-(trimethylsilyloxy)-1-arylmethylphosphonates instead of 1-amino-1-arylmethylphosphonate (Scheme 1).

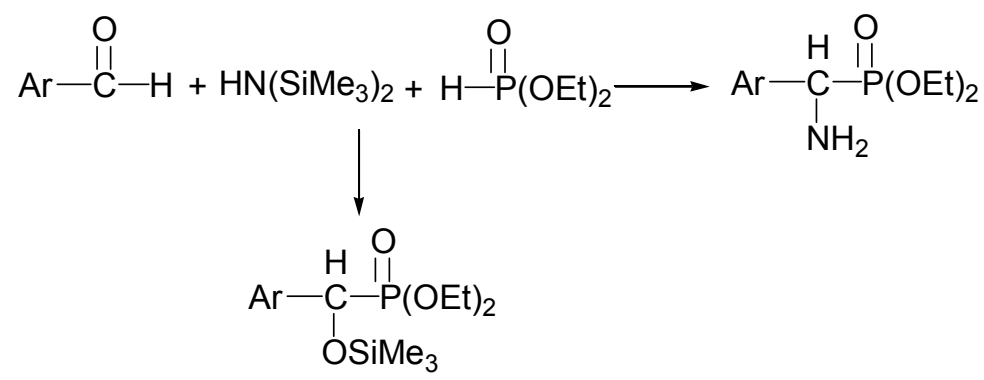

\section{Scheme 1}

They believed that HMDS does not react with carbonyl compounds and dialkyl phosphate reacts with aldehydes (or ketones) to give 1-hydroxyalkylphosphonates. ${ }^{2}$ As part of our efforts to explore the utility of solid phase reactions for the synthesis of organophosphorus compounds, ${ }^{17}$ we decided to analyze and reinvestigate our reaction and comments on this reaction.

\section{Results and Discussion}

In contrast to Soroka and Kolodziejczyk's report ${ }^{2}$ that HMDS does not react with carbonyl compound we found that the reaction of benzaldehyde (1a), as model compound, with HMDS under solvent-free condition in the absence of alumina leads to the long-known substance " $N, N$ 'bis(phenylmethylidene) phenylmethane diamine (2a) as the sole product ( according to Scheme 2). ${ }^{18}$ As it has been shown in experimental section, the article published in $1997,{ }^{1}$ when HMDS and aromatic aldehydes was stirred for $15 \mathrm{~min}$ in the presence of acidic alumina, an exothermic reaction took place, which. Toru et al. ${ }^{19}$ publication has shown the products must be compounds 2. Consequently the same products $\mathbf{2}, N, N^{\prime}$-bis(arylmethylidene)-arylmethanediamines, are 
prepared from the reaction of aromatic aldehydes with HMDS under solvent-free condition in the presence or absence of acidic alumina. Then $N, N^{\prime}$-bis(arylmethylidene)arylmethane diamines (2) reacts with diethyl phosphite to give diethyl $N$-arylidene-1-amino-1-arylmethylphosphonates (3), which can be easily hydrolyzed to diethyl-1-amino arylmethyl phosphonates and isolated as its sulphonate salt (4) (Scheme 2).

We examined usage of various types of alumina (acidic, basic and neutral) and also magnesia for the synthesis of 1-aminophosphonates. We found that the reaction of HMDS with benzaldehyde in the presence of diethyl phosphite using of acidic alumina gave diethyl $\mathrm{N}$ (phenylmethylene)-1-amino-1-phenylmethylphosphonate (3a) as the major product. The diethyl 1-hydroxy-1-phenylmethylphosphonate was obtained as the major product in the presence of magnesia. ${ }^{17 a}$ A 1:1 ratio of 3a and diethyl 1-hydroxy-1-phenylmethylphosphonate obtained by using of neutral or basic alumina.

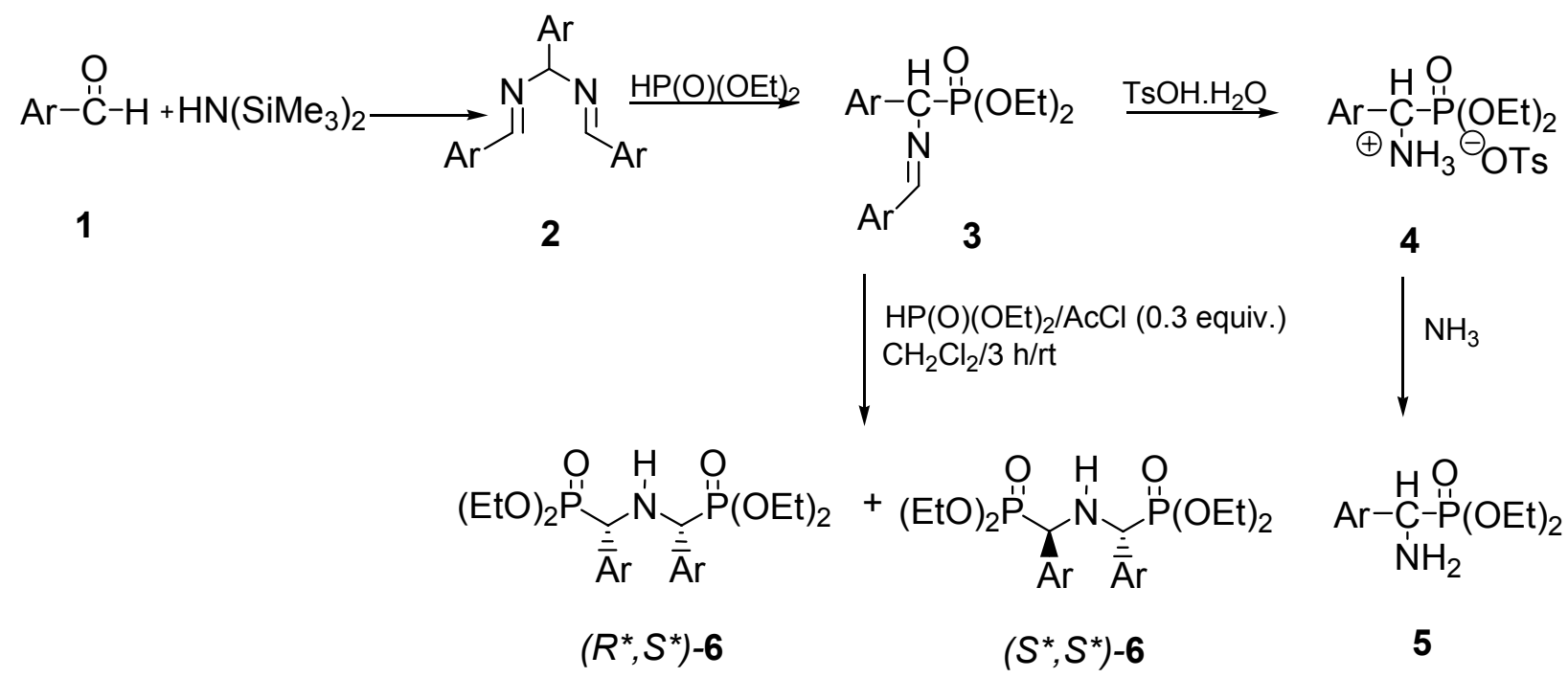

\section{Scheme 2}

It was found that the reaction of imine 3a with diethyl phosphite in the presence of catalytic amount of acetyl chloride give bis[1-diethoxyphosphorylphenyllmethyl] amine $\mathbf{6 a}$ as sole product in good yield and diastereomeric excess (Table 1). The product has been used as chelating agent for polyvalent metal ions, particularly alkaline earth metal ions. ${ }^{20}$ This process was successfully applied to other imines $\mathbf{3}$ as summarized in Table 1.

According to Scheme 2, diethyl N-arylmethylene-1-amino-1-arylmethylphophonates (3) react with diethyl phosphite and catalytic amount acetyl chloride to afford the desired products in good yields, 6b-6g in Table 1. It was suggested that in situ generation of $\mathrm{HCl}$ catalyzed this reaction.

In Summary, for the preparation of diethyl 1-amino-1-arylmethylphosphonate we recommended the reaction of aromatic aldehydes with HMDS followed by reaction with diethyl phosphate in the presence of alumina to give diethyl N-arylmethylene-1-amino-1- 
arylmethylphophonate (3), which can be easily hydrolyzed to a diethyl-1-aminoarylmethylphosphonates. Further hydrophosphonylation of imine $\mathbf{3}$ with diethyl phosphate catalyzed by acetyl chloride to afford bis[1-diethoxyphosphorylaryllmethyl] amine as sole product. A simple work-up, low consumption of solvent, fast reaction rates, mild reaction conditions, good yields, relatively clean reactions with no tar formation make our method as an attractive and a useful contribution to present methodologies.

Table 1. Reaction of imine 3 with diethylphosphite in the presence of catalytic amount of acetyl chloride

\begin{tabular}{|c|c|c|c|c|c|}
\hline \multirow[t]{2}{*}{ Entry } & \multirow{2}{*}{$\begin{array}{l}\mathrm{Ar} \\
\mathbf{3}\end{array}$} & \multirow{2}{*}{$\begin{array}{l}\text { Yield } \%{ }^{\mathrm{a}} \\
6\end{array}$} & \multicolumn{2}{|c|}{${ }^{31} \mathrm{P}$ NMR $(\oint \mathrm{ppm})$} & \multirow[t]{2}{*}{ de $\%{ }^{b}$} \\
\hline & & & major & minor & \\
\hline $\mathbf{a}$ & $\mathrm{C}_{6} \mathrm{H}_{5^{-}}$ & 65 & 23.36 & 23.68 & 63 \\
\hline b & $p-\mathrm{MeOCHC}_{6} \mathrm{H}_{4}-$ & 60 & 23.78 & 24.00 & 56 \\
\hline c & $p-\mathrm{ClC}_{6} \mathrm{H}_{4-}$ & 61 & 22.53 & 22.90 & 42 \\
\hline d & $p-\mathrm{FC}_{6} \mathrm{H}_{4-}$ & 70 & 22.30 & 22.67 & 46 \\
\hline $\mathbf{e}$ & $p-\mathrm{CH}_{3} \mathrm{C}_{6} \mathrm{H}_{4-}$ & 73 & 23.69 & 23.97 & 51 \\
\hline $\mathbf{f}$ & $m-\mathrm{FC}_{6} \mathrm{H}_{4-}$ & 63 & 21.71 & 22.17 & 26 \\
\hline g & $\beta$-Naphthyl & 57 & 23.27 & 23.60 & 42 \\
\hline
\end{tabular}

${ }^{a}$ Yield refers to isolated yield by column chromatography; ${ }^{b}$ Diastereomeric excess (de) was calculated by ${ }^{31} \mathrm{P}$ NMR spectrum.

\section{Experimental Section}

General Procedures. All chemicals were commercial products and distilled or recrystallized before use. NMR spectra were taken with a 250 Brucker Avance instrument with the chemical shifts being reported as $\delta$ ppm and couplings expressed in Hertz. The chemical shift data for each signal on ${ }^{1} \mathrm{H}$ NMR are given in units of $\delta$ relative to $\mathrm{CHCl}_{3}(\delta=7.26)$ for $\mathrm{CDCl}_{3}$ solution. For ${ }^{13} \mathrm{C}$ NMR spectra, the chemical shifts in $\mathrm{CDCl}_{3}$ and DMSO are recorded relative to the $\mathrm{CDCl}_{3}$ resonance $(\delta=77.0)$. The chemical shifts of ${ }^{31} \mathrm{P}$ are recorded relative to external $85 \% \mathrm{H}_{3} \mathrm{PO}_{4}$ $(\delta=0)$ with broad-band ${ }^{1} \mathrm{H}$ decoupling. Silica gel column chromatography was carried out with Silica gel 100 (Merck No. 10184). Merck Silica-gel 60 F254 plates (No. 5744) were used for the preparative TLC.

Synthesis of $N, N^{\prime}$-bis(phenylmethylidene) phenylmethanediamine from the reaction of benzaldehyde with HMDS under solvent-free condition. Benzaldehyde (1.06 g, $10 \mathrm{mmol})$ and HMDS (1.93 g, $12 \mathrm{mmol})$ were stirred for $5 \mathrm{~h}$ at $60{ }^{\circ} \mathrm{C}$. A solid was formed. $n$-Hexane $(10 \mathrm{~mL})$ was added to this mixture, filtered, washed with dry $n$-hexane, and dried under vacuum to afford $0.80 \mathrm{gr}(80 \%)$ of colorless solid: $\mathrm{mp} 101-102{ }^{\circ} \mathrm{C}\left(\mathrm{CH}_{2} \mathrm{Cl}_{2} / n\right.$-hexane) (lit. $\left.{ }^{20} \mathrm{mp} 100-101{ }^{\circ} \mathrm{C}\right)$. 


\section{General procedure for the synthesis of compounds $5 \mathbf{a}-\mathrm{g}$ and $6 \mathbf{a - g}$}

Acidic alumina ( $1 \mathrm{~g})$ and HMDS (1.93 g, $10 \mathrm{mmol})$ were mixed at room temperature. Aromatic aldehyde $(10 \mathrm{mmol})$ was added dropwise to the mixture with stirring. After completion of aldehyde addition, acidic alumina ( 2 gr) was added while resultant mixture was stirred. An exothermic reaction took place at this step thus stirring of mixture was continued for 15 min until its temperature reached to room temperature. Diethyl phosphite $(1.38 \mathrm{gr}, 10 \mathrm{mmol})$ was added to the reaction vessel and the mixture was stirred for $2 \mathrm{~h}$. The reaction mixture was extracted with ether $(100 \mathrm{ml})$ :

Synthesis of 1-aminophosponic acid esters (5a-g). $p$-Ts $\mathrm{OH} . \mathrm{H}_{2} \mathrm{O}(1.9 \mathrm{~g}, 10 \mathrm{mmol})$ was added to ethereal solution and stirred for $3 \mathrm{hrs}$. The solid salt was filtrated and neutralized with $\mathrm{NH}_{4} \mathrm{OH}$ $(10 \%)$. Extraction with ether $(3 \times 50 \mathrm{ml})$, evaporation of solvent and chromatography on plug of silica gel with EtOAc/n-hexane (9:1) gave the pure product as oil in $42-65 \%$ yields. All products are known and gave satisfactory spectral data in accord with the assigned structures and literature reports. $^{21}$

Synthesis of bis[1-diethoxyphosphorylarylmethyl] amine (6a-g). Ethereal extract was concentrated and the residue was chromatographed on plug of silica gel with EtOAc/n-hexane $(1: 1)$ to give the pure product 3 . The product $3(3 \mathrm{mmol})$ was added to a mixture of diethylphosphite $(5 \mathrm{mmol})$ in dichloromethane $(10 \mathrm{ml})$. Acetyl chloride $(1 \mathrm{mmol})$ was added dropwise to reaction mixture. The reaction mixture was stirred for $3 \mathrm{~h}$ at room temperature. Evaporation of solvent and chromatography on plug of silica gel with ethyl acetate-methanol (9:1) and evaporation of the solvent under reduced pressure gave the pure product as colorless oil in $57-73 \%$ yields. All products are known and gave satisfactory spectral data in accord with the assigned structures and literature reports. ${ }^{22}$

Diethyl $\left\{[\right.$ (diethylphosphoryl) (phenyl) methyl] amino $\}$ (phenyl)methylphosphonate (6a). ${ }^{22}$ Colorless oil (65\%); ${ }^{1} \mathrm{H}-\mathrm{NMR}\left(\mathrm{CDCl}_{3} / \mathrm{TMS}-250 \mathrm{MHz}\right): 1.10(6 \mathrm{H}, \mathrm{t}, J=7.1 \mathrm{~Hz}), 1.26(6 \mathrm{H}, \mathrm{t}$, $J=7.1), 2.93$ (1H, br, -NH), 3.65-4.35 (10H, m), $7.29(10 \mathrm{H}, \mathrm{s}) .{ }^{31} \mathrm{P}-\mathrm{NMR}\left(\mathrm{CDCl}_{3} / \mathrm{H}_{3} \mathrm{PO}_{4}\right): 23.36$, 23.68. ${ }^{13} \mathrm{C}-\mathrm{NMR}\left(\mathrm{CDCl}_{3} / \mathrm{TMS}-62.9 \mathrm{MHz}\right): 134.3,128.8,128.2,128.1,62.6-62.9$ (c), 57.4 (dd, $J_{\mathrm{PC}}=155.1$ and $\left.17.9 \mathrm{~Hz}\right), 16.1-16.3(\mathrm{c})$.

Diethyl \{[(diethylphosphoryl) (p-methoxyphenyl) methyl] amino\}(p-methoxyphenyl)methyl phosphonate (6b). ${ }^{22 b, c}$

Colorless oil (60\%); ${ }^{1} \mathrm{H}-\mathrm{NMR}\left(\mathrm{CDCl}_{3} / \mathrm{TMS}-250 \mathrm{MHz}\right): 1.14(6 \mathrm{H}, \mathrm{t}, J=7.1 \mathrm{~Hz}), 1.29(6 \mathrm{H}, \mathrm{t}$, $J=7.1), 1.69$ (1H, br, -NH), $3.79(3 \mathrm{H}, \mathrm{s}) 3.68-4.35(10 \mathrm{H}, \mathrm{m}), 6.87$ (4H, d, $J=8.5 \mathrm{~Hz}), 7.22(4 \mathrm{H}, \mathrm{d}$, $\mathrm{J}=8.5 \mathrm{~Hz}) .{ }^{31} \mathrm{P}-\mathrm{NMR}\left(\mathrm{CDCl}_{3} / \mathrm{H}_{3} \mathrm{PO}_{4}\right): 23.78,24.00 ;{ }^{13} \mathrm{C}-\mathrm{NMR}\left(\mathrm{CDCl}_{3} / \mathrm{TMS}-62.9 \mathrm{MHz}\right): 159.4$, $129.9,125.9,113.8,62.4-62.8(\mathrm{c}), 56.4\left(\mathrm{dd}, J_{\mathrm{PC}}=156.7\right.$ and $\left.17.9 \mathrm{~Hz}\right), 55.1,16.1-16.3$ (c).

Diethyl \{[(diethylphosphoryl) (p-chlorophenyl) methyl] amino\}(p-chlorophenyl)methylphosphonate (6c). ${ }^{22 b, c}$

Colorless oil (61\%); ${ }^{1} \mathrm{H}-\mathrm{NMR}\left(\mathrm{CDCl}_{3} / \mathrm{TMS}-250 \mathrm{MHz}\right): 1.15(6 \mathrm{H}, \mathrm{t}, J=7.1 \mathrm{~Hz}), 1.29(6 \mathrm{H}, \mathrm{t}$, $J=7.1), 2.19$ (1H, br, -NH), 3.65-4.35 (10H, m), $7.23(4 \mathrm{H}, \mathrm{d}, \mathrm{J}=8.25 \mathrm{~Hz}), 7.33(4 \mathrm{H}, \mathrm{d}, \mathrm{J}=8.25$ $\mathrm{Hz}) .{ }^{31} \mathrm{P}-\mathrm{NMR}\left(\mathrm{CDCl}_{3} / \mathrm{H}_{3} \mathrm{PO}_{4}\right): 22.53,22.90 ;{ }^{13} \mathrm{C}-\mathrm{NMR}\left(\mathrm{CDCl}_{3} / \mathrm{TMS}-62.9 \mathrm{MHz}\right): 134.1,132.9$, $130.1,128.9,62.9-63.2(\mathrm{c}), 56.9\left(\mathrm{dd}, J_{\mathrm{PC}}=155.4\right.$ and $\left.17.6 \mathrm{~Hz}\right), 16.3-16.4$ (c). 
Diethyl \{[(diethylphosphoryl)(p-fluorophenyl)methyl] amino\}(p-fluorophenyl)methylphosphonate (6d). ${ }^{22 b, c}$ Colorless oil (70\%); ${ }^{1} \mathrm{H}-\mathrm{NMR}\left(\mathrm{CDCl}_{3} / \mathrm{TMS}-250 \mathrm{MHz}\right): 1.04(6 \mathrm{H}, \mathrm{t}$, $J=7.0 \mathrm{~Hz}), 1.18(6 \mathrm{H}, \mathrm{t}, J=7.0 \mathrm{~Hz}), 2.82(1 \mathrm{H}, \mathrm{br},-\mathrm{NH}), 3.57-4.22(10 \mathrm{H}, \mathrm{m}), 6.80-6.97$ (4H, m), 7.10-7.22 (4H, m). ${ }^{31} \mathrm{P}-\mathrm{NMR}\left(\mathrm{CDCl}_{3} / \mathrm{H}_{3} \mathrm{PO}_{4}\right): 22.30,22.67 .{ }^{13} \mathrm{C}-\mathrm{NMR}\left(\mathrm{CDCl}_{3} / \mathrm{TMS}-62.9 \mathrm{MHz}\right)$ : $162.0\left(\mathrm{~d}, J_{\mathrm{CF}}=246.5 \mathrm{~Hz}\right), 130.4,115.7,115.3,62.6-63.0(\mathrm{c}), 56.6\left(\mathrm{dd}, J_{\mathrm{PC}}=156.6\right.$ and $\left.17.6 \mathrm{~Hz}\right)$, 16.1-16.3 (c).

Diethyl \{[(diethylphosphoryl) (p-methylphenyl) methyl] amino\}(p-methylphenyl)methylphosphonate (6e). ${ }^{22 b, c}$ Colorless oil (73\%); ${ }^{1} \mathrm{H}-\mathrm{NMR}\left(\mathrm{CDCl}_{3} / \mathrm{TMS}-250 \mathrm{MHz}\right): 1.04$ (6H, t, $J=7.1$ $\mathrm{Hz}), 1.19$ (6H, t, J=7.1), 2.24 (3H, s), 2.85 (1H, br, -NH), 3.60-4.25 (10H, m), 6.95-7.15 (8H, m). ${ }^{31} \mathrm{P}-\mathrm{NMR}\left(\mathrm{CDCl}_{3} / \mathrm{H}_{3} \mathrm{PO}_{4}\right): 23.69,23.97 .{ }^{13} \mathrm{C}-\mathrm{NMR}\left(\mathrm{CDCl}_{3} / \mathrm{TMS}-62.9 \mathrm{MHz}\right): 137.7,131.1$, 129.2, 128.7, 62.4-62.9 (c), 57.0 (dd, $J_{\mathrm{PC}}=156.0$ and $\left.18.2 \mathrm{~Hz}\right), 21.1,16.1-16.3(\mathrm{c})$.

Diethyl $\{[$ (diethylphosphoryl) (m-fluorophenyl) methyl] amino\}(m-fluorophenyl)methylphosphonate (6f). ${ }^{22 b, c}$ Colorless oil (63\%); ${ }^{1} \mathrm{H}-\mathrm{NMR}\left(\mathrm{CDCl}_{3} / \mathrm{TMS}-250 \mathrm{MHz}\right): 1.11$ (6H, t, $J=7.0$ $\mathrm{Hz}), 1.24$ (6H, t, J=7.0 Hz), 2.92 (1H, br, -NH), 3.65-4.25 (10H, m), 6.85-7.09 (6H, m), 7.15$7.32(2 \mathrm{H}, \mathrm{m}) .{ }^{31} \mathrm{P}-\mathrm{NMR}\left(\mathrm{CDCl}_{3} / \mathrm{H}_{3} \mathrm{PO}_{4}\right): 21.71,22.17 .{ }^{13} \mathrm{C}-\mathrm{NMR}\left(\mathrm{CDCl}_{3} / \mathrm{TMS}-62.9 \mathrm{MHz}\right)$ : $162.9\left(\mathrm{~d}, J_{\mathrm{CF}}=246.8 \mathrm{~Hz}\right), 137.1,130.1,124.5,115.7,115.3,62.9-63.2$ (c), 57.2 (dd, $J_{\mathrm{PC}}=155.1$ and $17.4 \mathrm{~Hz}), 16.1-16.3$ (c).

Diethyl \{[(diethylphosphoryl) $(\beta$-naphthyl) methyl] amino\}( $\beta$-naphthylphenyl)methyl phosphonate (6g). ${ }^{22 \mathrm{~b}, \mathrm{c}}$ Colorless oil (57\%); ${ }^{1} \mathrm{H}-\mathrm{NMR}\left(\mathrm{CDCl}_{3} / \mathrm{TMS}-250 \mathrm{MHz}\right): 1.10(6 \mathrm{H}, \mathrm{t}$, $J=7.0 \mathrm{~Hz}), 1.31(6 \mathrm{H}, \mathrm{t}, J=7.0), 3.03(1 \mathrm{H}, \mathrm{br},-\mathrm{NH}), 3.70-4.57(10 \mathrm{H}, \mathrm{m}), 7.35-7.92(14 \mathrm{H}, \mathrm{m}) .{ }^{31} \mathrm{P}-$ NMR $\left(\mathrm{CDCl}_{3} / \mathrm{H}_{3} \mathrm{PO}_{4}\right): 23.27,23.60 .{ }^{13} \mathrm{C}-\mathrm{NMR}\left(\mathrm{CDCl}_{3} / \mathrm{TMS}-62.9 \mathrm{MHz}\right): 133.3,132.0,131.9$, $128.4,127.9,127.7,126.2,62.8-63.1$ (c), 57.7 (dd, $J_{\mathrm{PC}}=155.1$ and $\left.17.8 \mathrm{~Hz}\right), 16.1-16.3$ (c).

\section{Acknowledgements}

The Institute for Advanced Studies in Basic Sciences (IASBS) is thanked for supporting this work.

\section{References and Notes}

1. Sardarian, A. R.; Kaboudin, B. Tetrahedron Lett. 1997, 38, 2543.

2. Soroka, M.; Kolodziejczyk, K. Tetrahedron Lett. 2003, 44, 1863.

3. (a) Engel, R. Chem Rev. 1977, 77, 349. (b) Hiratake, J.; Oda, J. Biosci. Biotechnol. Biochem. 1997, 61, 211. (c) Schug, K. A.; Lindner. W. Chem. Rev. 2005, 105, 67. (d) Moonen, K.; Laureyn, I.; Stevens, C. V. Chem. Rev. 2004, 104, 6177. (e) Palacios, F.; Alonso, C.; de los Santos, J. M. Current Organic Chemistry 2004, 8, 1481. (f) Yamagishi, T.; Haruki, T.; Yokomatsu, T. Tetrahedron 2006, 62, 9210. (g) Yokomatsu, T.; Yamagishi, T.; Shibuya S. J. Chem. Soc., Perkin Trans. 1 1997, 1527. 
4. Kafarski, P.; Lejczak, B.; Tyka, R.; Koba, L.; Pliszczak, E.; Wieczorek, P. J. Plant Growth Regulation 1995, 14, 199.

5. (a) Giannousis, P. P.; Bartlett, P. A. J. Med. Chem. 1987, 30, 1603. (b) Maier, L.; Lea, P. J. Phosphorus Sulfur 1983, 17, 1. (c) Baylis, E. K.; Campbell, C. D.; Dingwall, J. G. J. Chem. Soc., Perkin Trans. 1. 1984, 2845. (d) Hilderbrand, R. L. In The Role of Phosphonates in Living Systems, CRC Press: Boca Raton, 1982. (e) Kafarski, P.; Lejczak, B. Phosphorus Sulfur 1991, 63, 193. (b) Kukhar, V. P.; Hudson, H. R. In Aminophosphonic and Aminophosphinic Acids, John Wiley \& Sons: New York, 2000.

6. (a) Hanessian, S.; Bennani, Y. L. Synthesis, 1994, 1272. (b) Redmore, D. In Topics in Phosphorus Chemistry; Griffith, E. J.; Grayson, M., Eds.; Vol. 8; Wiley: New York, 1976.

7. Atherton, F. R.; Hassall, C. H.; Lambert, R. W. J. Med. Chem., 1986, $29,29$.

8. Allen, M. C.; Fuhrer, W.; Tuck, B.; Wade, R.; Wood, J. M. J. Med. Chem. 1989, 32, 1652.

9. Hassal, C. H., In Antibiotics; Hahn, F. E., Ed.; Springer Verlag: Berlin, 1983; Vol VI, 1-11.

10. (a) Bhagat, S.; Chakraborti, A. K. J. Org. Chem. 2007, 72, 1263. (b) Kaboudin, B. Phosphorus, Sulfur and Silicon 2002, 177, 1749.

11. Gancarz, R.; Wieczorek, J. S. Synthesis, 1978, 625.

12. Seyferth, D.; Marmor, R. S.; Hilbert, P. J. Org. Chem. 1971, 36, 1379.

13. Worms, K. H.; Schmidt-Dunker, M. In Organic Phosphorus Compounds, Kosolapoff, G. M.; Marier, L., Eds., John Wiley \& Sons: New York, 1976: Vol. 7, p 1.

14. Barycki, J.; Mastalerz, P.; Soroka, M. Tetrahedron Lett. 1970, 36, 3147.

15. (a) Genet, J. P.; Uziel, J.; Port, M.; Touzin, A. M.; Roland, S.; Thorimbert, S.; Tanier, S. Tetrahedron Lett. 1992, 33, 77. (b) Qian, C.; Huang, T. J. Org. Chem. 1998, 63, 4125. (c) Ranu, B. C.; Hajira, A.; Jana, U. Org. Lett. 1999, 1, 1141. (d) Manabe, K.; Kobayashi, S. Chem. Commun. 2000, 669. (e) Chandrasekhar, S.; Prakash, S. J.; Jagadeshwar, V.; Narsihmulu, C. Tetrahedron Lett. 2001, 42, 5561.

16. (a) Naseem, A.; van Lier, J. E. Tetrahedron Lett. 2007, 48, 13. (b) Villemin, D.; Cheikh, N.; Mostefa-Kara, B.; Bar, N.; Choukchou-Braham, N.; Didi, M. A. Tetrahedron Lett. 2006, 47, 5519. (c) Kaboudin, B. Karimi, M. Biorg. Med. Chem. Lett. 2006, 16, 5324. (d) Huang, X.; Liu, J.; Chen, J.; Xu, Y.; Shen, W. Catal. Lett. 2006, 108, 79. (e) Kaboudin, B.; Elhamifar, D.; Farjadian, F. Org. Prep. Proced. Int. 2006, 38, 412. (f) Gauvin, R. M.; Mortreux, A. Chem. Commun. 2005, 1146 (g) Kaboudin, B.; Norouzi, H. Synthesis 2004, 2035. (h) Shimizu, K.-I.; Hayashi, E.; Hatamachi, T.; Kodama, T.; Kitayama, Y. Tetrahedron Lett. 2004, 45, 5135. (i) Dongare, M. K.; Bhagwat, V. V.; Ramana, C. V.; Gurjar, M. K. Tetrahedron Lett. 2004, 45, 4759. (j) Balakrishna, M. S.; Kaboudin, B. Tetrahedron Lett. 2001, 42, 1127. (k) Song, Y.-M.; Choi, J. S.; Yang, J. W.; Han, H. Tetrahedron Lett. 2004, 45, 3301. (1) Kaboudin, B.; Norouzi, H. Tetrahedron Lett. 2004, 45, 1283. (m) Kaboudin, B.; Saadati, F. J. Heterocycl. Chem. 2005, 42, 699. (n) Kaboudin, B.; Navaee, K. Heterocycles 2003, 60, 2287. (o) Danks, T. N.; Desai, B. Green Chem. 2002, 4, 179.

17. (a) Sardarian, A. R.; Kaboudin, B. Synth. Commun 1997, 27, 543. (b) Kaboudin, B. Chem. Lett. 2001, 880. (c) Kaboudin, B.; Nazari, R. Tetrahedron Lett. 2001, 42, 8211. (d) 
Kaboudin, B.; Nazari, R. Synth. Commun. 2001, 31, 2245. (e) Kaboudin, B.; Balakrishna, M. S. Synth. Commun. 2001, 31, 2773. (f) Kaboudin, B. Tetrahedron Lett. 2002, 43, 8713. (g) Kaboudin, B. Tetrahedron Lett. 2003, 44, 1051. (h) Kaboudin, B.; Rahmani, A. Synthesis 2003, 2705. (i) Kaboudin, B.; Saadati, F. Synthesis 2004. 1249. (j) Kaboudin, B.; Rahmani, A. Org. Prep. Proced. Int. 2004, 36, 82. (k) Kaboudin B.; Moradi, K. Tetrahedron Lett. 2005, 46, 2989. (1) Kaboudin, B.; Haghighat, H. Tetrahedron Lett. 2005, 46, 7955. (m) Kaboudin, B.; Haghighat, H.; Yokomatsu, T. J. Org. Chem. 2006, 71, 6604. (n) Kaboudin, B.; Karimi, M. Bioorg. Med. Chem. Lett. 2006, 16, 5324. (o) Kaboudin, B.; Farjadian, F. Beilstein J. Org. Chem. 2006, 2:4.

18. Reddy, P. Y.; Shimizu, M.; Higashi, K.; Shimizu, T.; Toru, T. Arkivoc 2001, 11.

19. (a) Uchida, H.; Shimizu, T.; Reddy, P. Y.; Nakamura, S.; Toru, T. Synthesis 2003, 1236. (b) Uchida, H.; Tanikoshi, H.; Nakamura, S.; Reddy, P. Y.; Toru, T. Synlett 2003, 1117.

20. (a) Berworth, F. C. U. S. Patent 2,599,807, 1952; Chem. Abstr. 1953, 47, 4360b. (b) Banks, C. V.; Yerick, R. E. Anal. Chim. Acta 1959, 20, 301.

21. (a) Lukszo, J.; Kowalik, J.; Mastalerz, P., Chem. Lett. 1978, 1103. (b) Green, D.; Geeta, P.; Elgendy, S.; Baban, J. A.; Claeson, G.; Kakkar, V. V.; Deadman, J., Tetrahedron, 1994, 50, 5099. (c) Berry, J. P.; Isbell, A. F.; Hunt, G. E., J. Org. Chem., 1972, 26, 4396. (d) Dzygiel, P.; Rudzinska, E.; Wieczorek, P.; Kafarski, P., Journal of Chromatography A 2000, 895, 301 and references cited their.

22. (a) Dimukhametov, M. N.; Musin, R. Z.; Buzykin, B. I.; Latypov, S. K.; Mironov, V. F. Mendeleev Communications 2005, 40. (b) Kaboudin, B.; Moradi, K. Synthesis 2006, 2339. (c) Kaboudin, B.; Jafari, E. Synthesis 2006, 3063. 